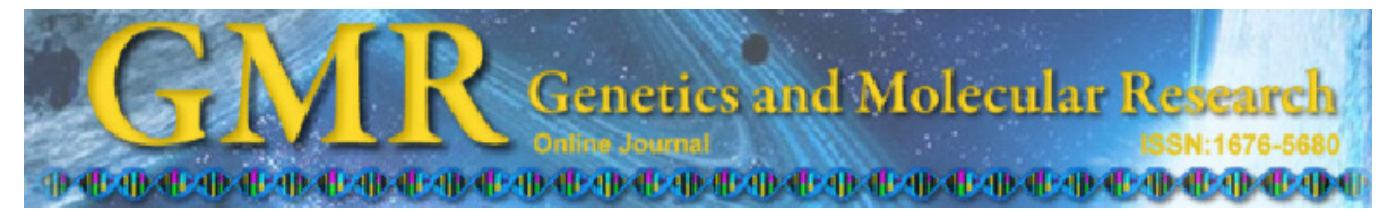

\title{
Meta-analysis demonstrates association of the TGF- $\beta 1$ gene -C509T polymorphism with susceptibility to IgA nephropathy in European but not in Asian populations
}

\author{
H. Wang ${ }^{1,2}$, P. $\mathrm{Li}^{2}$ and Z.-C. Feng ${ }^{1}$ \\ ${ }^{1}$ School of Medicine and Health Management, \\ HuaZhong University of Science and Technology, Wuhan, China \\ ${ }^{2}$ First Affiliated Hospital, Anhui Medical University, Hefei, Anhui, China \\ Corresponding author: Z.-C. Feng \\ E-mail: zhanchunfengcn@163.com
}

Genet. Mol. Res. 12 (1): 434-442 (2013)

Received June 11, 2012

Accepted October 22, 2012

Published February 8, 2013

DOI http://dx.doi.org/10.4238/2013.February.8.8

\begin{abstract}
There are conflicting reports associating the TGF- $\beta 1$ gene -C509T polymorphism with susceptibility to IgA nephropathy. We investigated this association through a meta-analysis. Case-control studies were searched up to January 2012; the genotype frequencies in the control group were found to be consistent with Hardy-Weinberg equilibrium. Publication bias was tested by funnel plot and the Egger regression test. Eight studies, comprising 1364 cases and 1483 controls, were included. Significant heterogeneity was observed $\left(\chi^{2}=18.29, \mathrm{P}\right.$ $=0.01)$. Under the random-effects model, the overall odds ratio (OR) was $1.01[95 \%$ confidence interval $(95 \% \mathrm{CI})=0.74-1.38 ; \mathrm{P}=0.94]$. In the subgroup analysis based on ethnicities, no significant effect was observed in the Asian descent groups (five comparisons, $\mathrm{OR}=0.78$; $95 \% \mathrm{CI}=0.53-1.15$; moderate heterogeneity between studies). However, an association was observed in the European descent groups $(\mathrm{OR}=1.5$; $95 \% \mathrm{CI}=1.15-1.96 ; \mathrm{P}=0.003$; no significant heterogeneity between studies). There was no evidence of publication bias according to funnel plot and the Egger regression test $(\mathrm{a}=-2.16, \mathrm{P}=0.23)$. There was
\end{abstract}


heterogeneity between studies and no clear evidence of an association between the TGF- $\beta 1$ gene -C509T polymorphism and susceptibility to $\operatorname{IgA}$ nephropathy in the worldwide population. Subgroup analysis suggests that the TGF- $\beta 1$ gene -C509T polymorphism would not be a risk factor for IgA nephropathy in Asians but might play a role in Europeans. More studies are required for definitive conclusions.

Key words: IgA nephropathy; -C509T polymorphism; TGF- $\beta 1$ gene; Meta-analysis

\section{INTRODUCTION}

Immunoglobulin A nephropathy (IgAN), the most common glomerulonephritis throughout the world, exhibits an indolent but slowly progressive course leading to end-stage renal failure in 30 to $40 \%$ of patients older than 30 years (Brezzi et al., 2009). Its variable clinical picture likely depends on the diverse interplay of environmental and genetic factors in each patient. During the past decade, several studies have investigated the polymorphisms of the genes related to molecules in the renin-angiotensin system - interleukin (IL)-1, IL-6 and IL-10 (Bantis et al., 2004), IL-1 receptor, tumor necrosis factor alpha, uteroglobin, T-cell receptors, and nephrin (Narita et al., 2003; Kovacs et al., 2006) - and data have emerged to support the hypothesis that genetic factors affect susceptibility to both the onset and progression of IgAN (Narita et al., 2002; Syrjanen et al., 2002; Wiwanitkit, 2006; Yamamoto et al., 2012).

Members of the transforming growth factor- $\beta$ (TGF- $\beta$ ) family play a crucial role in the pathogenesis of renal fibrosis thanks to their prosclerotic activity exerted by inducing cell proliferation and extracellular matrix molecule deposition. TGF- $\beta$ and its respective receptors are abnormally expressed in experimental and human nephropathies (Julian et al., 2007; Bhowmik et al., 2011). TGF- $\beta 1$ is a TGF- $\beta$ isoform that is highly expressed during the onset and progression of various renal diseases including IgAN (Song et al., 2003; Hohenstein et al., 2008; Iwano, 2010; Lee, 2011). Furthermore, a close relationship between increased renal tissue levels of TGF- $\beta 1$ and fibronectin, one of the major TGF- $\beta 1$-regulated extracellular components, has been reported in IgAN patients (Lee, 2011). The TGF- $\beta 1$ gene is located on chromosome $19 \mathrm{q} 13$ and is highly polymorphic. Five polymorphisms in white populations have been identified: 2 in the promoter region at positions -800 and $-509,1$ at position +72 in a non-translated region, and 2 in the signal sequence at positions +869 and +915 . Among these polymorphisms, -C509T is reportedly associated with some clinical phenotypes (Awad et al., 1998; Baan et al., 2000; Yokota et al., 2000; Yamada et al., 1998, 2001; Lacha et al., 2001). Grainger et al. (1999) have found that the C-509T polymorphism is associated with the circulating concentration of TGF- $\beta 1$, which is significantly lower in white women with the CC genotype compared with that in subjects with other genotypes.

The function and location of the TGF- $\beta 1$-C509T polymorphism make it a solid candidate for association with IgAN, so a candidate gene analysis of TGF- $\beta 1-C 509 \mathrm{~T}$ was recently performed. Functional variants of the gene encoding TGF- $\beta 1$-C509T were reportedly associated with IgAN. In addition, Vuong et al. (2009) have reported an association between the TGF- $\beta 1$-C509T polymorphism and IgAN. Lim et al. (2005) and Qin et al. (2008) have found a similar association. Thus, the TGF- $\beta 1-C 509 \mathrm{~T}$ susceptibility gene has an important role in the pathogenesis of IgAN. 
Despite the association between TGF- $\beta 1$-C509T and IgAN observed in some Swedish, South Korea, and Chinese populations (Lim et al., 2005; Vuong et al., 2009; Li et al., 2011), the results of later studies are inconsistent (Carturan et al., 2004; Sato et al., 2004; Xue et al., 2005; Qin et al., 2008; Brezzi et al., 2009). Considering the possible small effect size of this genetic polymorphism in IgAN and the relatively small sample size in each study, discrepancies are likely to become apparent because a single study may have been underpowered for the detection of a small but real association.

Given the amount of accumulated data now available, a quantitative synthesis of the evidence using rigorous methods is important. The aim of this study was to assess the association of the TGF- $\beta 1$-C509T polymorphism with the risk of IgAN by conducting a metaanalysis of individual datasets from all eligible case-control studies published to date.

\section{MATERIAL AND METHODS}

\section{Data}

PubMed, the Chinese Biomedical Database (CBM), Chinese National Knowledge Infrastructure, and Wanfang (Chinese) were searched for genetic association studies published before January 2012 that evaluated the TGF- $\beta 1$-C509T polymorphism and IgAN in humans. We also reviewed the reference lists of relevant articles and performed Baidu and Google searches to identify additional studies. The PubMed search was run using the mesh terms ["Glomerulonephritis, IgA" (Mesh), "IgA nephropathy", "IgAN"], and ("TGF-1" or "TGF" or "TGF- $\beta 1$-C509T" or "transforming growing factor"). In the Chinese Biomedical Database, China National Knowledge Infrastructure, and Wanfang, the following words were used: ("TGF- $\beta 1$ " or "Chinese technical term of TGF- $\beta 1$ ") and ("IgA nephropathy" or "glomerulonephritis" or "relevant Chinese technical terms").

\section{Study selection}

The following criteria were used to identify relevant published studies: 1) the studies used case-control or cohort designs that determined the distribution of the C677T and A1298C genotypes in cancer-free subjects and subjects with gastric cancer diagnosed with histopathological biopsy, 2) Ethics Committee approval and informed patient consent were obtained, 3) the studies provided relative risk estimates and respective variance or the relevant information needed to calculate it, and 4) the distribution of the genotypes in control groups was in HardyWeinberg equilibrium (HWE), and 5) raw data were available for retrieval. When multiple publications were found for the same population, only the latest or largest study was included. Meeting abstracts, case reports, editorials, and review articles were excluded.

\section{Data extraction and synthesis}

Data were independently extracted by 2 reviewers (Heng Wang and Peng Li) using a standardized data extraction form. Discrepancies were resolved through discussion, and if consensus was not reached, the decision was made by a third reviewer (Zhan-chun Feng). The title and abstract of all potentially relevant articles were screened to determine their relevance. 
Full articles were also scrutinized if the title and abstract were ambiguous. The following information was collected from each study: first author's surname, year of publication, ethnicity, criteria of enrolled patients, study design, total number of cases and controls, and number of cases and controls with the -C509T polymorphism (TT vs CT and CC genotypes).

\section{Statistical analysis}

Because case-control studies were involved, odds ratio (ORs) were used to assess the strength of the association between the TGF- $\beta 1$-C509T polymorphism and IgAN. We calculated the ORs and respective $95 \%$ confidence intervals (95\% CIs) by comparing the carriers of rare alleles with the wild homozygote as CT + CC vs TT.

The chi-square test was used to check for HWE of genotypes in the control group of each study. Statistical heterogeneity among studies was assessed with Q and $\mathrm{I}^{2}$ statistics (Higgins and Thompson, 2002). $\mathrm{I}^{2}$ values of 25,50 , and $75 \%$ were considered to be low, moderate, and high estimates, respectively. Heterogeneity was considered to be significant at a $\mathrm{P}$ value of $<0.10$. Lower heterogeneity indicated a more credible result.

A fixed-effects model using the Mantel-Haenszel method and a random-effects model using the DerSimonian and Laird method were used to pool the results (Petitti, 1994). Random effects are more appropriate when heterogeneity is present compared with the fixed-effects model that was used without heterogeneity. The significance of the pooled OR was determined using the Z-test and considered to be significant at a $\mathrm{P}$ value of $<0.05$.

Publication bias was investigated using a funnel plot, in which the standard error of $\log (\mathrm{OR})$ of each study was plotted against its OR. An asymmetric plot suggested possible publication bias. Funnel plot asymmetry was assessed with the Egger linear regression test method, which uses a linear regression approach to measure funnel plot asymmetry on the natural logarithm scale of the OR (Egger et al., 1997). The result was credible when publication bias was absent.

Analyses were performed using Stata version 10 (StataCorp LP, College Station, TX, USA), Review Manager 4.2 (Cochrane Collaboration, http://www.cc-ims.net/RevMan). All P values were 2 sided. A $P$ value of $<0.05$ was considered to be statistically significant.

\section{RESULTS}

\section{Subject characteristics}

Eight association studies relating to the TGF- $\beta 1$ gene -C509T polymorphism with susceptibility to IgAN met the inclusion requirements for the meta-analysis (Carturan et al., 2004; Sato et al., 2004; Lim et al., 2005; Xue et al., 2005; Qin et al., 2008; Brezzi et al., 2009; Vuong et al., 2009; Li et al., 2011). A total of 1364 cases and 1483 controls were investigated. Three studies were from China (Xue et al., 2005; Qin et al., 2008; Li et al., 2011), 2 from Italy (Carturan et al., 2004; Brezzi et al., 2009), 1 from Japan (Sato et al., 2004), 1 from Korea (Lim et al., 2005), and 1 from Sweden (Vuong et al., 2009). All cases of IgAN were diagnosed using World Health Organization diagnosis criteria. Selected characteristics of the 8 case-control studies of the relationship between the TGF- $\beta 1$ gene-C509T polymorphism and susceptibility to IgAN are summarized in Table 1. 
Table 1. Characteristics of published studies about the association between the TGF- $\beta 1$-C509T polymorphism with immunoglobulin A nephropathy included in the meta-analysis and results of Hardy-Weinberg test for genotype distribution of the TGF- $\beta 1$-C509T polymorphism in control groups of reviewed studies.

\begin{tabular}{|c|c|c|c|c|c|c|c|c|}
\hline \multirow[t]{3}{*}{ Investigator } & \multirow[t]{3}{*}{ Year } & \multirow[t]{3}{*}{ Country } & \multirow[t]{3}{*}{ Ethnicity } & \multicolumn{4}{|c|}{ TGF- $\beta 1-509 \mathrm{C} / \mathrm{T}$ polymorphism } & \multirow[t]{3}{*}{ HWE P } \\
\hline & & & & \multicolumn{2}{|c|}{ Case } & \multicolumn{2}{|c|}{ Control } & \\
\hline & & & & $\mathrm{CC}+\mathrm{CT}$ & TT & $\mathrm{CC}+\mathrm{CT}$ & TT & \\
\hline Carturan et al. & 2004 & Italy & European & 84 & 11 & 83 & 21 & 0.193 \\
\hline Li et al. & 2011 & China & Asian & 27 & 11 & 32 & 10 & 0.768 \\
\hline Vuong et al. & 2009 & Sweden & European & 121 & 88 & 223 & 245 & 0.711 \\
\hline Xue et al. & 2005 & China & Asian & 319 & 68 & 164 & 38 & 0.879 \\
\hline Lu et al. & 2008 & China & Asian & 79 & 40 & 93 & 23 & 0.776 \\
\hline Sato et al. & 2004 & Japan & Asian & 263 & 66 & 233 & 64 & 0.31 \\
\hline Lim et al. & 2005 & Korea & Asian & 49 & 33 & 42 & 13 & 0.34 \\
\hline Brezzi et al. & 2009 & Italy & European & 82 & 23 & 146 & 54 & 0.481 \\
\hline
\end{tabular}

HWE $=$ Hardy-Weinberg equilibrium.

The genotype frequencies in the control group were consistent with HWE (Table 2). The distribution of ORs from individual studies with respect to their standard deviation was symmetrical in the funnel plot (Figure 1). The Egger test was performed to provide statistical evidence of funnel plot symmetry $(\mathrm{a}=-2.16, \mathrm{t}=-1.31, \mathrm{P}=0.237$; Figure 2$)$. These data provided no significant evidence of publication bias.

Table 2. Egger linear regression test for publication bias of the IgAN gene TGF- $\beta 1$-C509T polymorphism $(\mathrm{a}=$ $-2.16, \mathrm{t}=-1.31, \mathrm{P}=0.23$ for $\mathrm{C}$ allele $v$ s $\mathrm{T}$ allele).

\begin{tabular}{|c|c|c|c|c|c|c|}
\hline Egger test (standard efficiency) & Coefficient & Standard error & $\mathrm{t}$ & $\mathrm{p}>\mathrm{t} \mid$ & \multicolumn{2}{|c|}{$95 \%$ Confidence interval } \\
\hline Slope & 0.6235989 & 0.422415 & 1.48 & 0.190 & -4.100134 & 1.657211 \\
\hline Bias & -2.158793 & 1.642344 & -1.31 & 0.237 & -6.177464 & 1.859879 \\
\hline
\end{tabular}

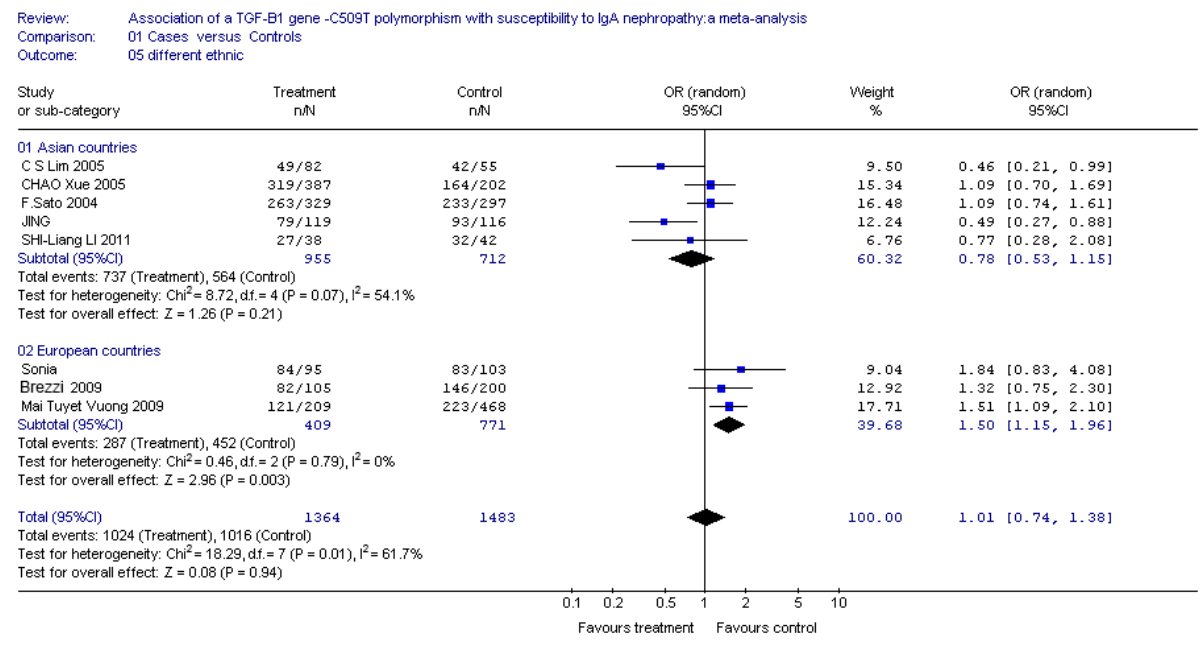

Figure 1. Funnel plot of 8 studies and relationship between TGF- $\beta 1-C 509 \mathrm{~T}$ and IgAN to determine publication bias. $\mathrm{x}$-axis $=\log$ odds ratio (OR) of the TGF- $\beta 1$-C509T allele; $\mathrm{y}$-axis $=$ standard error of $\log$ OR; d.f. $=$ degrees of freedom; $95 \% \mathrm{CI}=95 \%$ confidence interval. 


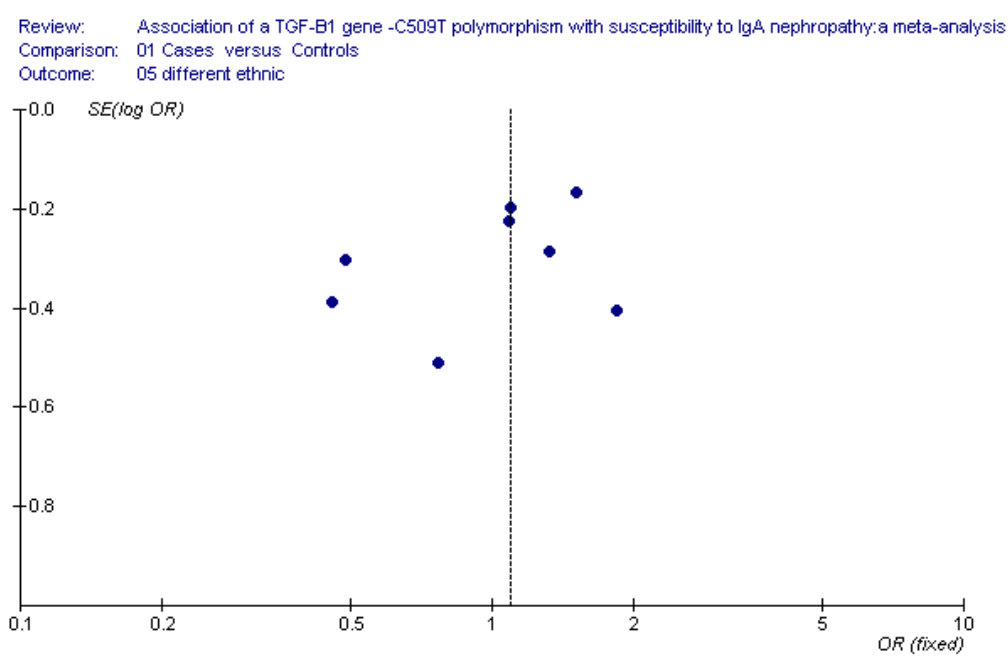

Figure 2. Egger linear regression test for publication bias of the IgAN gene TGF- $\beta 1-C 509 \mathrm{~T}$ polymorphism $(\mathrm{a}=$ $-2.16, \mathrm{t}=-1.31, \mathrm{P}=0.23$ for $\mathrm{C}$ allele $v s \mathrm{~T}$ allele). $\mathrm{SE}=$ standard error; $\mathrm{OR}=$ odds ratio.

\section{Association between TGF-ß1 -C509T and IgAN}

Heterogeneity was observed among individual estimates of the ORs (chi-square $=$ $18.29, \mathrm{P}=0.01$ ), and the original data were combined by means of the random-effects model. Statistics calculated for each study are shown in a forest plot (Figure 3). The summary OR was $1.01(95 \% \mathrm{CI}=0.74-1.38 ; \mathrm{P}=0.94)$ according to the random-effects model. No evidence was found to suggest that the TGF- $\beta 1$ gene-C509T polymorphism increased susceptibility to IgAN in a worldwide population.

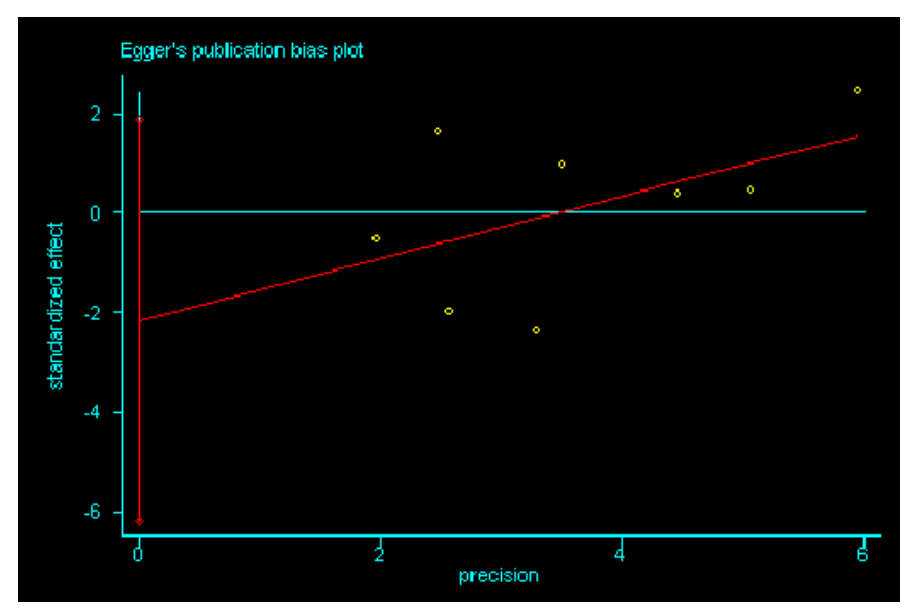

Figure 3. Overall and pooled odds ratio (ORs) with $95 \%$ confidence interval $(95 \% \mathrm{CI})$ for overall analysis and subgroup analysis testing association of the TGF- $\beta 1$-C509T polymorphism with IgAN. The aggregate OR and $95 \% \mathrm{CI}$ of the risk allele are also given. The weighting factors (weight \%) used to calculate the aggregate OR, calculated from the inverse of the variance, are given for each study. 
A previous report was published on the association between TGF- $\beta 1$ T869C and rheumatoid arthritis and revealed discrepancies that may have been due to ethic differences. What is meant by "discrepancies on the association?" What kinds of discrepancies? To look for an ethnic effect, we performed a subgroup meta-analysis in populations of Asian and European decent. The results indicated that the TGF- $\beta 1$ gene -C509T polymorphism had no effect on susceptibility to IgAN in subgroups of Asian descent ( 5 comparisons; OR $=0.78 ; 95 \% \mathrm{CI}=$ 0.53-1.15; moderate between-study heterogeneity) under the random-effects model. However, an association was observed in the European subgroup under the fixed-effect models $(\mathrm{OR}=$ $1.5 ; 95 \% \mathrm{CI}=1.15-1.96 ; \mathrm{P}=0.003$; no significant between-study heterogeneity; see Figure 3 ).

\section{DISCUSSION}

Since the first positive association reported between the TGF- $\beta 1$ gene-C509T polymorphism and susceptibility to IgAN in a Japanese population, 7 other studies have been undertaken to replicate the association. However, subsequent studies in other populations have been inconsistent. Therefore, we did a meta-analysis to estimate the relationship between the TGF- $\beta 1$ gene-C509T polymorphism and susceptibility to IgAN. Our meta-analysis is the first to examine this relationship. Overall, the results showed that the TGF- $\beta 1$ gene-C509T polymorphism may not be an IgAN susceptibility gene across populations. The common OR for the risk allele was $1.01(95 \% \mathrm{CI}=0.74-1.38 ; \mathrm{P}=0.94)$. An association was found between the TGF- $\beta 1$ gene -C509T polymorphism and IgAN in European, but not Asian, populations in the subgroup meta-analysis.

Several explanations are possible for varying roles played by the same polymorphism in different ethnic populations or across different studies. First, the existence of the genetic heterogeneity results from the geographical environment and living habits of subject, which influence the frequency distribution of the crowd gene. Some studies have shown that the difference in C509T gene distribution between European and Asian countries is statistically significant (Cotton et al., 2002; Holla et al., 2002; Chen et al., 2005). Second, the clinical characteristics of participants - for example, age and years from onset, male predominance - may lead to different outcomes. Sometimes association can only be found in stratification analysis according to clinical characters. In this study, such information could not be obtained completely. Moreover, only 8 studies were included, which is inconvenient for subgroup studies. These influences may be the main source of the reported heterogeneity in this article.

The focus of meta-analysis is to combine comparable studies to increase sample size and statistical power and draw more compelling results. However, meta-analysis has confounding factors such as publication bias, diverse genetic backgrounds of subjects, and varying data-gathering protocols, methods of sampling, and quality analyses. A funnel plot and the Egger linear regression test used to assess publication bias suggested that bias was absent. Testing HWE for distribution of the genotypes in control groups also suggested that no significantly different genetic background was present among the participants. We followed the inclusion and exclusion criteria strictly according to standard protocols. The 8 studies appear to be comparable related to the meta-analysis.

Nevertheless, the analysis has several limitations. First, the number of total samples in the meta-analysis is small, and the amount of ethnic stratification in the subgroup analyses is even smaller. The amount of gender stratification is too small to conduct the subgroup analysis 
by gender. More studies are required to draw definite conclusions. Second, heterogeneity exists between studies, possibly owing to covariates: age and years from onset, gender, fraction of patients among these studies, and so on. Third, the selection of control participants in casecontrol studies may influence the results because hospital-based controls may not be as representative as population-based normal controls, although no evidence of an effect of the control population was detected in this meta-analysis. Considering the limited studies and population numbers included in our study, the results should be interpreted with caution.

\section{CONCLUSIONS}

Despite these limitations, this meta-analysis suggests that heterogeneity occurred between studies and found no clear evidence of an association between the TGF- $\beta 1$ gene-C509T polymorphism and susceptibility to IgAN in a worldwide population. Subgroup analysis results suggested that TGF- $\beta 1$ gene -C509T would not be a risk factor for IgAN in Asians but might play a role in IgAN susceptibility in Europeans. Because the number of studies included was relatively small, studies with larger pools of data are required for definitive conclusions.

\section{ACKNOWLEDGMENTS}

Research supported by a grant from Philosophy Social Science Planning Project of Anhui Province (\#2010AHSK09-10D108).

\section{REFERENCES}

Awad MR, El-Gamel A, Hasleton P, Turner DM, et al. (1998). Genotypic variation in the transforming growth factorbeta1 gene: association with transforming growth factor-beta1 production, fibrotic lung disease, and graft fibrosis after lung transplantation. Transplantation 66: 1014-1020.

Baan CC, Balk AH, Holweg CT, van Riemsdijk IC, et al. (2000). Renal failure after clinical heart transplantation is associated with the TGF-beta 1 codon 10 gene polymorphism. J. Heart Lung Transplant. 19: 866-872.

Bantis C, Heering PJ, Aker S, Klein-Vehne N, et al. (2004). Association of interleukin-10 gene G-1082A polymorphism with the progression of primary glomerulonephritis. Kidney Int. 66: 288-294.

Bhowmik D, Sinha S, Gupt A, Tiwari SC, et al. (2011). Clinical approach to rapidly progressive renal failure. J. Assoc. Physicians India 59: 38-41.

Brezzi B, Del Prete D, Lupo A, Magistroni R, et al. (2009). Primary IgA nephropathy is more severe in TGF-beta1 high secretor patients. J. Nephrol. 22: 747-759.

Carturan S, Roccatello D, Menegatti E, Di Simone D, et al. (2004). Association between transforming growth factor beta1 gene polymorphisms and IgA nephropathy. J. Nephrol. 17: 786-793.

Chen BP, Wei YS, Xie JS and Huang HL (2005). Transforming growth factor beta1 gene polymorphisms in ZHUANG and HAN nationality in Guangxi Province. Chin. J. Anat. 28: 379-380.

Cotton SA, Gbadegesin RA, Williams S, Brenchley PE, et al. (2002). Role of TGF-beta1 in renal parenchymal scarring following childhood urinary tract infection. Kidney Int. 61: 61-67.

Egger M, Davey SG, Schneider M and Minder C (1997). Bias in meta-analysis detected by a simple, graphical test. BMJ 315: 629-634.

Grainger DJ, Heathcote K, Chiano M, Snieder H, et al. (1999). Genetic control of the circulating concentration of transforming growth factor type beta1. Hum. Mol. Genet. 8: 93-97.

Higgins JP and Thompson SG (2002). Quantifying heterogeneity in a meta-analysis. Stat. Med. 21: 1539-1558.

Hohenstein B, Daniel C, Wittmann S and Hugo C (2008). PDE-5 inhibition impedes TSP-1 expression, TGF-beta activation and matrix accumulation in experimental glomerulonephritis. Nephrol. Dial. Transplant. 23: 3427-3436.

Holla LI, Fassmann A, Benes P, Halabala T, et al. (2002). 5 polymorphisms in the transforming growth factor- $\beta 1$ gene (TGF-beta1) in adult periodontitis. J. Clin. Periodontol. 29: 336-341. 
Iwano M (2010). EMT and TGF-beta in renal fibrosis. Front Biosci. 2: 229-238.

Julian BA, Wyatt RJ, Matousovic K, Moldoveanu Z, et al. (2007). IgA nephropathy: a clinical overview. Contrib. Nephrol. 157: 19-26.

Kovacs TJ, Harris S, Vas TK, Seres I, et al. (2006). Paraoxonase gene polymorphism and serum activity in progressive IgA nephropathy. J. Nephrol. 19: 732-738.

Lacha J, Hubacek JA, Potmesil P, Viklicky O, et al. (2001). TGF-beta I gene polymorphism in heart transplant recipients - effect on renal function. Ann. Transplant. 6: 39-43.

Lee HS (2011). Pathogenic role of TGF-beta in the progression of podocyte diseases. Histol. Histopathol. 26: 107-116.

Li SL, Lin X, Wang J, Yang F, et al. (2011). Relationship of transforming growth factor $\beta 1$ gene $-509 \mathrm{C} / \mathrm{T}$ polymorphism with IgA nephropathy in Western Guangxi. China J. Modern Med. 21: 1607-1610.

Lim CS, Kim YS, Chae DW, Ahn C, et al. (2005). Association of C-509T and T869C polymorphisms of transforming growth factor-beta1 gene with susceptibility to and progression of IgA nephropathy. Clin. Nephrol. 63: 61-67.

Narita I, Saito N, Goto S, Jin S, et al. (2002). Role of uteroglobin G38A polymorphism in the progression of IgA nephropathy in Japanese patients. Kidney Int. 61: 1853-1858.

Narita I, Goto S, Saito N, Song J, et al. (2003). Genetic polymorphism of NPHS1 modifies the clinical manifestations of Ig A nephropathy. Lab. Invest. 83: 1193-1200.

Petitti DB (1994). Meta-Analysis, Decision Analysis, and Cost-Effectiveness Analysis. Oxford University Press, New York.

Qin W, Zhang YJ, Tan CY, Liu XR, et al. (2008). Association of TGF- $\beta 1$ gene polymorphism with IgA nephropathy. West China Med. J. 23: 61-63.

Sato F, Narita I, Goto S, Kondo D, et al. (2004). Transforming growth factor-beta1 gene polymorphism modifies the histological and clinical manifestations in Japanese patients with IgA nephropathy. Tissue Antigens 64: 35-42.

Song JH, Lee SW, Suh JH, Kim ES, et al. (2003). The effects of dual blockade of the renin-angiotensin system on urinary protein and transforming growth factor-beta excretion in 2 groups of patients with IgA and diabetic nephropathy. Clin. Nephrol. 60: 318-326.

Syrjanen J, Hurme M, Lehtimaki T, Mustonen J, et al. (2002). Polymorphism of the cytokine genes and IgA nephropathy. Kidney Int. 61: 1079-1085.

Vuong MT, Lundberg S, Gunnarsson I, Wramner L, et al. (2009). Genetic variation in the transforming growth factor- $\beta 1$ gene is associated with susceptibility to IgA nephropathy. Nephrol. Dial. Transplant. 24: 3061-3067.

Wiwanitkit V (2006). Angiotensin-converting enzyme gene polymorphism is correlated to the progression of disease in patients with IgA nephropathy: a meta-analysis. Ren. Fail. 28: 697-699.

Xue C, Li YJ, Li CX, Du Y, et al. (2005). Relationship of TGF- $\beta 1$-509C/T polymorphism with IgA nephropathy in HAN Nationality of Chinese Population. J. Sun Yat-Sen Univ. 26: 260-263.

Yamada Y, Miyauchi A, Goto J, Takagi Y, et al. (1998). Association of a polymorphism of the transforming growth factor-beta1 gene with genetic susceptibility to osteoporosis in postmenopausal Japanese women. J. Bone Miner. Res. 13: 1569-1576.

Yamada Y, Miyauchi A, Takagi Y, Tanaka M, et al. (2001). Association of the C-509 $\rightarrow$ T polymorphism, alone of in combination with the T869 $\rightarrow$ C polymorphism, of the transforming growth factor-betal gene with bone mineral density and genetic susceptibility to osteoporosis in Japanese women. J. Mol. Med. 79: 149-156.

Yamamoto R, Nagasawa Y, Shoji T, Katakami N, et al. (2012). A candidate gene approach to genetic contributors to the development of IgA nephropathy. Nephrol. Dial. Transplant. 27: 1020-1030.

Yokota M, Ichihara S, Lin TL, Nakashima N, et al. (2000). Association of a T29 $\rightarrow$ C polymorphism of the transforming growth factor-betal gene with genetic susceptibility to myocardial infarction in Japanese. Circulation 101: 27832787. 